\title{
The Development of Paeonia ostii Seeds and Oil Quality Formation
}

\author{
Yuting Zou, Yanan Wang, Mingwei Zhu, and Shuxian Li \\ Collaborative Innovation Center of Sustainable Forestry in Southern China, \\ College of Forest Science, Nanjing Forestry University, Nanjing 210037, \\ China
}

\author{
Qiuyue Ma \\ Institute of Leisure Agriculture in Jiangsu Academy of Agricultural Sciences, \\ Nanjing 210014, China
}

Additional index words. magnetic resonance imaging, oil body, seed development, unsaturated fatty acid

\begin{abstract}
Paeonia ostii is a woody oil crop with potential value as an edible oil. With the aim of acquiring systematic knowledge of the development of $P$. ostii seeds, the oil content, biomass, and water content of the seeds were determined. Changes in the distribution of hydrogen protons in $P$. ostii seeds during follicle development were traced using magnetic resonance imaging (MRI). The formation of oil bodies in the endosperm and embryo was observed using transmission electron microscopy (TEM). Dynamic changes in oleic acid, linoleic acid, and $\alpha$-linolenic acid contents were assessed by gas chromatography-mass spectrometry (GC-MS). The magnetic resonance images showed that, during early follicle development [45-85 days after flowering (DAF)], a greater quantity of liquid mucus was present within the seeds, and seeds in the same follicle developed at different rates. At 95 to $115 \mathrm{DAF}$, proton density was distributed evenly in all areas of the seed. A small dark area appeared in the center of the seed, and mucus in the follicles and water in the pericarp disappeared gradually. TEM observations showed that at $45 \mathrm{DAF}$, a few oil bodies were scattered at the cell periphery in the endosperm, and oil bodies were more numerous in the embryo. With the progression of seed development, the number and size of oil bodies in the embryo and endosperm continued to increase. The fresh and dry mass of $P$. ostii seeds increased from 45 to 105 DAF, then decreased after 105 DAF. The moisture content decreased, whereas the oil content increased and attained $33.1 \%$ at seed maturity. The three predominant unsaturated fatty acids accumulated simultaneously and showed stages of initial accumulation (45-65 DAF) and rapid accumulation (65-105 DAF). The results suggest that 65 to 105 DAF is a critical period for unsaturated fatty acid accumulation in $P$. ostii seeds.
\end{abstract}

Seeds serve as a strong metabolic library, which is why seed development is an important stage for higher plants. During the process of seed maturation, substances such as starch, protein, and lipids are transformed and accumulated. Pavithra et al. (2014) observed that oil content in Pongamia pinnata seeds increased with seed maturation, and oleic acid content determined by GC-MS remained high at the mature

Received for publication 10 Apr. 2020. Accepted for publication 26 May 2020.

Published online 26 June 2020.

We acknowledge funding received from the Province Graduate Research and Practice Innovation of Jiangsu for the project "Study on the mechanism of oil accumulation during the development of Paeonia ostii seeds" (KYCX18-0961).

We thank Robert McKenzie, from Liwen Bianji, Edanz Group China, for editing the English of a draft of this manuscript.

S.L. is the corresponding author. E-mail: shuxianli@ njfu.com.cn.

This is an open access article distributed under the CC BY-NC-ND license (https://creativecommons. org/licenses/by-nc-nd/4.0/).
(UFAs). Among the UFAs, $\alpha$-linolenic acid is the predominant type, accounting for $42.7 \%$ of the total content, which is substantially greater than that of traditional oil crops, such as soybean, rapeseed, peanut, corn, and sunflower (Li et al., 2014). For this reason, peony seed oil is being developed as a novel source of woody edible oil and is widely produced in China. In addition, the flower of $P$. ostii has a unique taste and high nutritional value (Gai et al., 2013; Han et al., 2011; Yuan et al., 2011). P. ostii is also of considerable medicinal value. The root bark is a traditional Chinese medicine rich in nutrients and it has therapeutic effects. Because of the wide variety of uses, $P$. ostii has received increasing attention from the Chinese government for development as an emerging woody oil crop (Li, 2014).

Currently present, research on peony is focused predominantly on the flower color and oil contents. However, relevant research on the behavior of seed oil accumulation and changes in UFAs during seed development are extremely scarce. In our study, we investigated seed and follicle development of $P$. ostii 'Fengdan' using MRI. The formation of oil bodies was observed using TEM. The seed mass, together with the contents of water, oil, $\alpha$-linolenic acid, oleic acid, and linoleic acid in the seeds at different developmental stages were determined. Our aim was to acquire systematic knowledge of oil body formation and accumulation of UFAs during seed maturation, which is essential to the management of $P$. ostii individuals in cultivation to optimize seed oil content. Optimal management will not only improve $P$. ostii seed oil quality, but also increase the seed yield to a certain extent (Shi, 2016).

\section{Materials and Methods}

stage. Yuan et al. (2015) reported that palmitic acid, stearic acid, and oleic acid contents of Camelina sativa seeds are high during the early stage of seed development, but the oil content is low. However, the oil content in the seeds accumulated rapidly during the intermediate stage of seed development and increased further during the final stage of seed development. Zhang et al. (2017) reported a linear decline in water content during seed development in Styrax tonkinensis. They also observed that oil accumulation fluctuated throughout seed development, and the contents of oleic acid and linoleic acid increased during the early stage of seed development. In contrast, oleic acid content increased slowly and linoleic acid content decreased slightly during the final stage of seed development.

Paeonia suffruticosa, the tree peony, is a famous traditional flower in China (Cheng and $\mathrm{Yu}, 2008)$. Paeonia ostii, commercially referred to as 'Fengdan', is a perennial deciduous shrub belonging to section Moutan of Paeonia, and is the most widely used species because of the high oil content of the seeds, $90.0 \%$ of which is unsaturated fatty acids
Plant material. We marked 100 six-yearold trees of $P$. ostii for collection of fruit samples, which were propagated from the seeds of Heze, Shandong Province, China (lat. $35^{\circ} 14^{\prime} \mathrm{N}$, long. $115^{\circ} 26^{\prime} \mathrm{E}$ ). The trees were planted in the field of the Nanjing Green Universe Peony Science \& Technology Co. Ltd, Jiangsu Province, China (lat. $32^{\circ} 11^{\prime}-32^{\circ} 27^{\prime} \mathrm{N}$, long. $118^{\circ} 34^{\prime}-119^{\circ} 03^{\prime} \mathrm{E}$ ), with $60-\mathrm{cm}$ spacing between plants and $80-\mathrm{cm}$ spacing between rows. This area belongs to the subtropical, monsoon climate zone, with mild climate and four distinctive seasons. The annual average precipitation, temperature, and accumulated temperature of the experimental site are $1004.4 \mathrm{~mm}, 16^{\circ} \mathrm{C}$, and $4820{ }^{\circ} \mathrm{C}$, respectively.

All chosen individuals bore five subbranches, blossomed on the same day of 6 most identical. Forty tagged follicles on the marked trees were collected randomly at 20-d intervals from 45 to $85 \mathrm{DAF}$, and then at $10-\mathrm{d}$ intervals until follicle maturity. After collection, a complete fresh fruit with five follicles was used for MRI analysis. About 80 seeds were extracted Apr. 2019, and the flower sizes were al- 
manually from the remaining follicles for measurement of seed biomass and analysis of moisture and oil contents, as well as TEM observation. The extracted seeds were stored in sealed, zippered plastic bags at $-80{ }^{\circ} \mathrm{C}$.

MRI analysis. MRI was performed using a MesoMR23-60H-I NMR imager (Suzhou Niumag Company Ltd, Suzhou, China) with a resonance frequency of $23.423 \mathrm{MHz}$, a probe coil diameter of $60 \mathrm{~mm}$, and a magnet temperature of $32.00 \pm 0.01{ }^{\circ} \mathrm{C}$. For all images, fresh follicles were used and the acquisition parameters were as follows: field of view, $100 \times 100 \mathrm{~mm}$; repetition time, $500 \mathrm{~ms}$; echo time, $20 \mathrm{~ms}$; slice thickness, $13.6 \mathrm{~mm}$; number of sampling times, 32; and matrix, $256 \times 256$.

Oil body observation by TEM. Fresh seeds from each sample were used to examine oil body formation using TEM. The seeds were cut vertically. Samples of the endosperm and embryo were prepared following the method described by Zhang et al. (2018), with some modifications. Briefly, the samples were fixed immediately with $2.5 \%(\mathrm{v} / \mathrm{v})$ glutaraldehyde in sodium phosphate buffer ( $\mathrm{pH} 7.2)$. The samples were postfixed in osmium tetroxide and dehydrated in ethyl alcohol at $4{ }^{\circ} \mathrm{C}$. The samples were embedded in Spurr's resin and observed at $60 \mathrm{kV}$ with a JEM-1200 transmission electron microscope (JEOL, Tokyo, Japan).

Seed moisture content, and fresh and dry weight analysis. The fresh and dry weight, as well as the moisture content of the seeds were estimated for three replicates of 20 seeds. The seeds were cut into small pieces of less than $2 \mathrm{~mm}$ and were weighed to $0.001-\mathrm{g}$ accuracy to obtain the fresh weight for each harvest. The seeds were then placed in a freezedryer (Christ Alpha 1-4 LOC-1; Martin Christ, Osterode, Germany) with a pressure of $0.001 \mathrm{MPa}$ at an initial temperature of $-76{ }^{\circ} \mathrm{C}$, followed by precooling at $-80{ }^{\circ} \mathrm{C}$ for $2 \mathrm{~h}$. After lyophilization for 24 $\mathrm{h}$, the samples attained a constant weight. The samples were then placed in a desiccator with silica gel. After the samples had returned to room temperature, they were reweighed to determine the dry weight. The moisture content at each sampling time point was calculated and expressed as the percentage weight loss relative to the initial weight.

Determination of seed oil content. Seed oil was extracted in accordance with the method described by Zhang et al. (2017). Twenty seeds with three replicates at each developmental stage were cut into small pieces and freeze-dried at $-76{ }^{\circ} \mathrm{C}$ until a constant mass was attained. The tissue pieces were ground to a powder and $1 \mathrm{~g}$ of powder was weighed. The oil was extracted with petroleum ether $\left(30\right.$ to $\left.60{ }^{\circ} \mathrm{C}\right)$ using a Soxhlet apparatus as described by Dutta et al. (2014). The flask temperature was maintained at $60{ }^{\circ} \mathrm{C}$ and the samples were extracted for $96 \mathrm{~h}$. After extraction, the powder was reweighed, and the amount of oil was expressed as the percentage weight loss relative to the initial dry weight per gram.

Measurement of UFA content by GC-MS. This experiment mainly followed the steps outlined by Zarringhalami et al. (2011), with some modifications. Fatty acid methyl esters were prepared by placing oil samples $(0.2 \mathrm{~g})$ extracted at different stages of seed development in screw-cap vials; $4 \mathrm{~mL}$ methanolic sodium hydroxide $(0.5 \mathrm{~mol} / \mathrm{L})$ was added to each vial. The vials were sonicated with an ultrasonic cleaner (KQ5200; Kunshan Ultrasonic Instrument Company, Suzhou, China) in a water bath at $60{ }^{\circ} \mathrm{C}$ until the oil droplets were dissolved completely. The vials were removed from the water bath and allowed to cool to room temperature. Next, $2 \mathrm{~mL}$ of $14 \%$ boron trifluoride was added to the vial as a catalyst, and the vials were sonicated in a water bath at $60{ }^{\circ} \mathrm{C}$ for $10 \mathrm{~min}$. After cooling to room temperature, saturated sodium chloride solution $(2 \mathrm{~mL})$ and hexane $(2 \mathrm{~mL})$ were added to the vials. The vials were corked and shaken thoroughly, then allowed to rest for 5 min. The upper hexane layer containing the methyl esters was collected and injected into a gas chromatograph (GC; Unicam 4600, Shimadzu, Kyoto, Japan).

The contents of oleic acid, linoleic acid, and $\alpha$-linolenic acid were determined using a GC equipped with a flame ionization detector (FID). The GC-MS conditions were as follows. A fused-silica capillary column (30-m $\times 0.25-\mathrm{mm}$ i.d. $\times 0.25-\mu \mathrm{m}$ film thickness) (TR-WAXMS; Thermo Scientific, Waltham, MA) was used. The GC split ratio was $1: 10$, and samples of about $0.5 \mu \mathrm{L}$ were injected. The FID and injector temperatures were set to $230{ }^{\circ} \mathrm{C}$ and $250^{\circ} \mathrm{C}$, respectively. The initial column temperature was maintained at $80^{\circ} \mathrm{C}$ for $2.5 \mathrm{~min}$ and increased to $210^{\circ} \mathrm{C}$ at a rate of $15{ }^{\circ} \mathrm{C} / \mathrm{min}$ and was held at $210{ }^{\circ} \mathrm{C}$. The temperature was then increased at a rate of $2{ }^{\circ} \mathrm{C} / \mathrm{min}$ until it attained $230{ }^{\circ} \mathrm{C}$ and was held for $10 \mathrm{~min}$. Helium was used as the carrier gas at a column head pressure of 20 psi. The sample peaks were identified by comparison with retention times for a pure standard mixture (Aldrich Chemical Company, Milwaukee, WI). The peak areas were calculated and the percentages of the areas were determined.

Statistical analysis. Descriptive statistics were derived using Excel 2010 (Microsoft Corporation, Redmond, WA). One-way analysis of variance was performed using IBM SPSS Statistics 22.0 (IBM Corporation, Armonk, NY) followed by Duncan's multiple range test for comparison of all pairs of treatment means. The $P$ values were considered to be significant within groups at the 0.05 significance level. The magnetic resonance images were analyzed using OsiriX Lite software (version 7.0.4; Pixmeo, Geneva, Switzerland) and Adobe Photoshop 7.0 (Adobe, San Jose, CA).

\section{Results}

Fresh and dry biomass of seeds. The fresh weight of $P$. ostii seeds increased rapidly from 45 to 65 DAF (Fig. 1). From 65 to 105 $\mathrm{DAF}$, the rate of increase in fresh weight declined slightly, but it maintained a linear upward trend, attaining a maximum fresh weight of $10.6 \mathrm{~g}$ (20 seeds, $105 \mathrm{DAF})$. Subsequently, a decline in fresh weight was observed at 115 DAF.

The dry weight showed a similar pattern to that of the fresh weight, with the weight increasing from 45 to 105 DAF. The maximum dry weight was $6.0 \mathrm{~g}$ at $105 \mathrm{DAF}$, from an initial weight of $1.1 \mathrm{~g}$. After $115 \mathrm{DAF}$, the dry weight decreased, but the decline was less remarkable than that observed for the fresh weight (Fig. 1).

Water and oil contents. The water and oil contents showed opposite trends during the development of $P$. ostii seeds (Fig. 2). The water content decreased significantly from $74.3 \%$ to $46.5 \%$ until 95 DAF. Subsequently, the water content maintained a slow decrease from $43.1 \%$ (105 DAF) to $41.6 \%$ (115 DAF); the decline was not significant. It is worth noting that $41.6 \%$ water content is extremely high compared with that of seeds of a majority of plant species.

The oil content showed a rapid increase from 45 to $85 \mathrm{DAF}$ - from $13.8 \%$ to $30.1 \%$. From 85 to $105 \mathrm{DAF}$, the oil content increased slightly. At $115 \mathrm{DAF}$, the oil content showed an abrupt increase, attaining the maximum value of $33.1 \%$.

MRI analysis of follicle development. MRI is capable of visualizing internal structural information to reflect the state of hydrogen protons (which are predominantly associated with water and oil in the samples) in a nondestructive manner (Prestes et al., 2007). The principle of MRI is that it uses static magnetic fields and radio frequencies to obtain images of proton mobility in biological systems. For a grayscale image, the greater the density of the hydrogen protons, the brighter the image (Mou et al., 2016). For a pseudocolor image, the color of each voxel represents the proton density, and colors from blue to bright yellow to red represent increased amounts of hydrogen protons in the tissues. The number of seeds in individual follicles, detected by MRI, differed considerably, varying from 4 to 13 . The proton density images of the developing $P$. ostii seeds also emphasize the area of interest where the water and oil protons are present.

At 45 DAF (Fig. 3A), the brightest region of the seed was the peripheral area near the seedcoat, whereas the central area of the endosperm was dark. The size of dark areas in the same follicle varied. Within the follicles, the locule was filled predominantly with liquid mucus, which was further confirmed by peeling the follicles. From the pseudocolor image (Fig. 3B), it was observed that the peripheral area of some seeds was bright red, but yellow for other seeds. The color of the central area was green. This led us to conclude that the proton density in the seed was greatest near the seedcoat and that seeds in the same follicle did not develop at the same rate. At 65 DAF (Fig. 3C), the entire seed was brighter than at $45 \mathrm{DAF}$ and the 


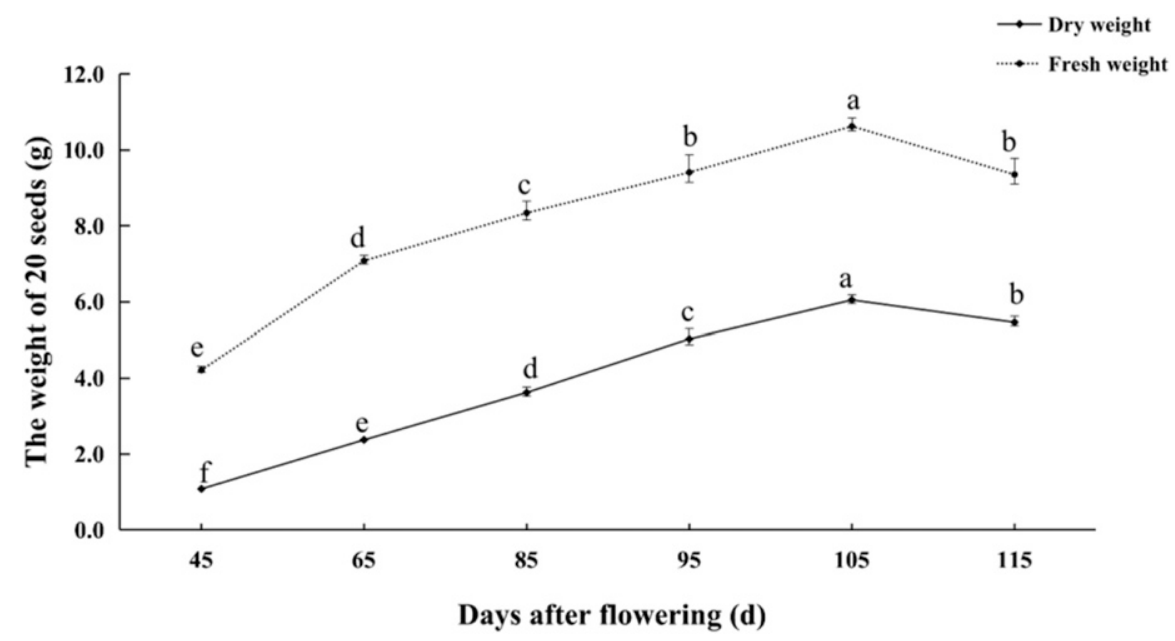

Fig. 1. Fresh and dry weight of Paeonia ostii seeds during follicle maturation. The different lowercase letters are significantly different according Duncan's multiple range test at $P \leq 0.05$.

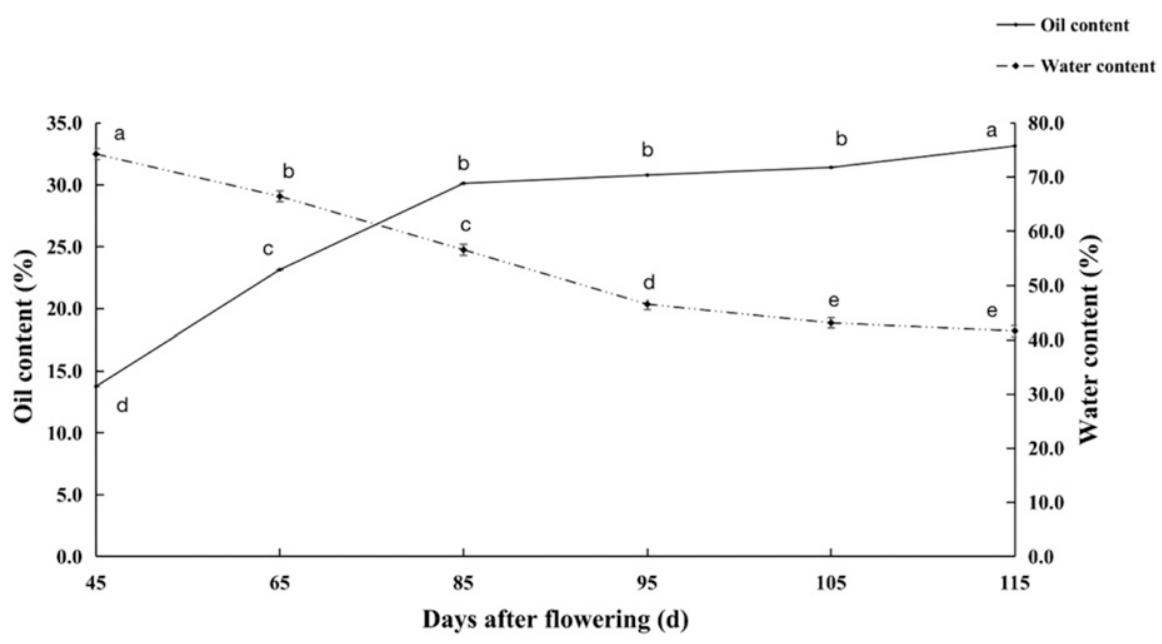

Fig. 2. Water and oil contents of Paeonia ostii seeds during follicle maturation. The different lowercase letters are significantly different according Duncan's multiple range test at $P \leq 0.05$.

dark space in the central area of the seed disappeared. However, a considerable volume of mucus was still present within the locule of the follicle. Based on the pseudocolor image (Fig. 3D), different colors were observed in different areas of the seed, and the developmental stage of the seeds still varied. At $85 \mathrm{DAF}$ (Fig. 3E), the brightness of the pericarp was less than observed previously and the seed shape had become irregular as a result of squeezing of the seeds, accompanied by a marked decrease in the amount of liquid mucus in the follicle. The internal development of the seed was completed at this stage. With maturation of the seeds (95 DAF), red areas were detected in almost all seeds in the follicles in the pseudocolor image (Fig. 3H), showing that proton density in $P$. ostii follicles had increased again. At 105 DAF (Fig. 3I), a small dark area (as shown by the red arrow) was observed in the center of the seed, and mucus in the follicle had completely disappeared. At 115 DAF (Fig. 3K), the coat of P. ostii follicles in some areas was extremely dark and the outline of the pericarp was indistinct, of oil bodies increased and the spaces between the oil bodies were reduced in size. By the time of $P$. ostii seed maturity (115 DAF), a large amount of tightly packed oil bodies almost filled the spaces between the highly electron-dense substances.

Compared with the images of the endosperm, no notable variation was observed in the images of the embryo except at $45 \mathrm{DAF}$. In the embryo at $45 \mathrm{DAF}$, the cell wall boundary was more distinct. Embryos were more active and accumulated more numerous oil bodies, and the size of the oil bodies was much larger, thus the formation of oil bodies preceded their formation in the endosperm. The images of the embryo also indicate that the cell walls became thicker with the progression of seed development.

UFA content during seed development. Oleic acid, linoleic acid, and $\alpha$-linolenic acid are the predominant UFAs present in $P$. ostii seeds. The accumulation of these three components showed the same trends (Fig. 5). The UFAs were almost absent before 65 DAF (content at 45 and 65 DAF, $<1.0 \%$ ), then increased in an almost linear manner from 65 to $105 \mathrm{DAF}$. At an advanced stage of maturation (after 105 DAF), oleic acid and $\alpha$-linolenic acid content plateaued, whereas linoleic acid content displayed an upward trend, slowly increasing by $3.7 \%$ during the last stage. The final contents of oleic acid, linoleic acid, and $\alpha$-linolenic acid were $23.2 \%$, $32.4 \%$, and $38.9 \%$, respectively.

\section{Discussion}

Accumulation of oil in P. ostii seeds. Extensive research on oil accumulation during seed development has been undertaken in a number of angiosperm species, including Pongamia pinnata (Pavithra et al., 2012) and Xanthoceras sorbifolium seeds (Zhao, 2010). All studies showed that the oil content increased gradually, whereas the water content decreased. However, in some species, such as sunflowers, arabidopsis, rapeseed, and sesame, the rapid accumulation of oil occurred at an advanced stage of seed development (Wiberg and Stymne, 1997). Oil accumulation in P. ostii seeds differed in that the rapid accumulation of oil was observed at earlier stages of development (before $85 \mathrm{DAF}$ ).

UFAs in P. ostii seeds. UFA components differ among oil crops, and their accumulation patterns also differ significantly. During stages of Pongamia pinnata seed maturation, Pavithra et al. (2012) observed a significant increase in oleic acid content, whereas linoleic acid content decreased from $30.14 \%$ to $18.85 \%$ and the $\alpha$-linolenic acid content remained constant, which differed from the pattern observed in $P$. ostii seeds. In our study, accumulation of the three predominant UFAs in P. ostii seeds showed a "slow-fast-slow" accumulation pattern, which was identical to that for oleic acid in Pongamia pinnata seeds. The dynamic changes in the three UFAs indicates it is necessary to enhance fertilizer management for $P$. ostii shrubs 65 to 105 DAF. 

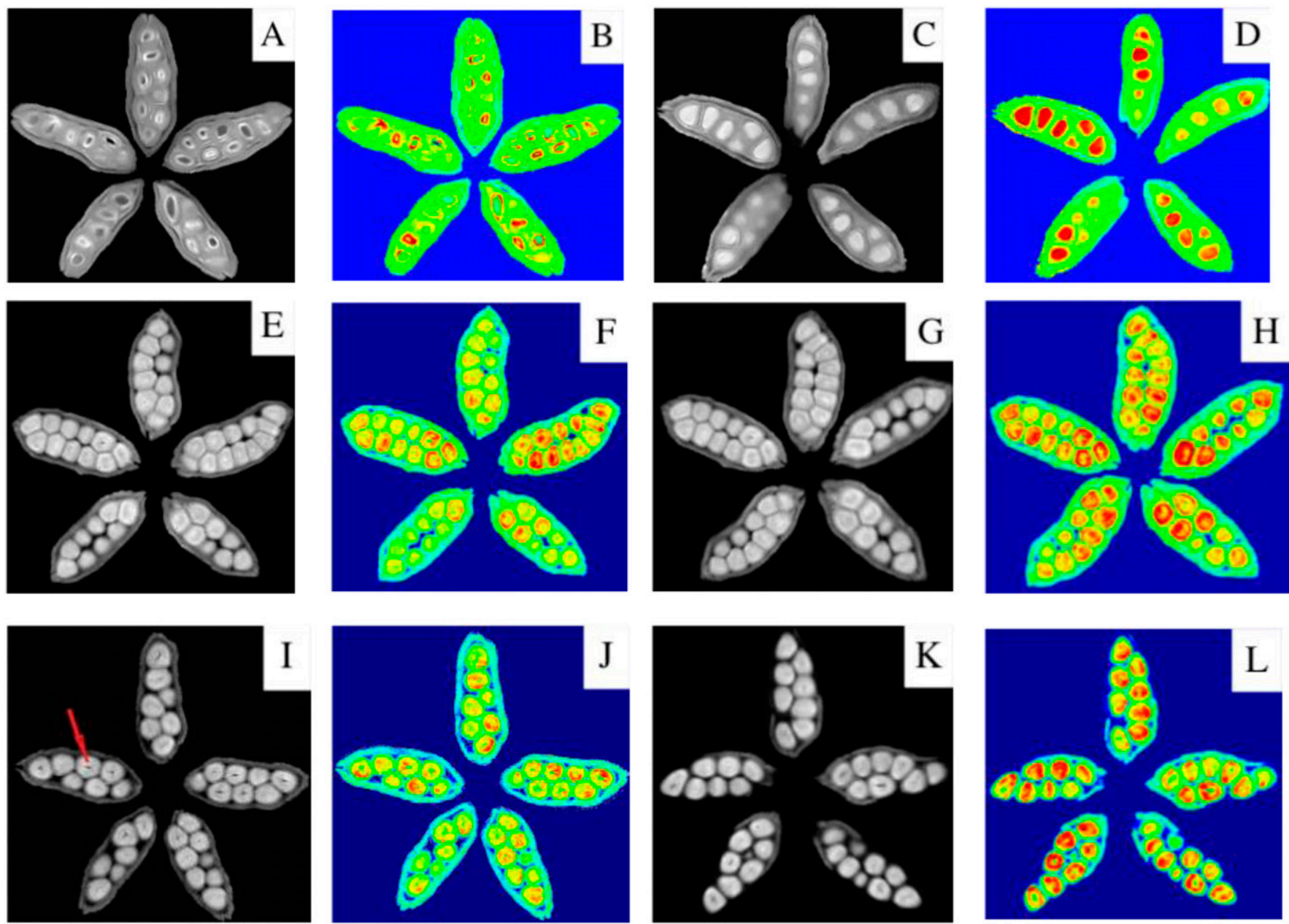

250

200

150

100

50

0

Fig. 3. Magnetic resonance images of Paeonia ostii follicles during maturation. Grayscale and pseudo-color images of follicles (A, B) $45 \mathrm{~d}$ after flowering (DAF), (C, D) $65 \mathrm{DAF},(\mathbf{E}, \mathbf{F}) 85 \mathrm{DAF},(\mathbf{G}, \mathbf{H}) 95 \mathrm{DAF},(\mathbf{I}, \mathbf{J}) 105 \mathrm{DAF}$, and $(\mathbf{K}, \mathbf{L}) 115 \mathrm{DAF}$. The color scale in the legend represents, from deep red to yellow to deep blue, a gradual decrease in proton density.

In our study, the oil content attained $23.1 \%$ at $65 \mathrm{DAF}$, but the content of the three UFAs in $P$. ostii seeds increased only slightly by less than $1 \%$. The reason may be that the accumulation of fatty acids was affected by a number of fatty acid synthetases (Fofana et al., 2006; Haralampidis et al., 1998) and the synthetases were not fully activated before 65 DAF; thus, the accumulation pathway of the three major UFAs was blocked. However, the precise reason warrants additional research.

TEM observations revealed the oil bodies present in the endosperm and embryo of $P$. ostii seeds were similar to those of the majority of plant species. At $65 \mathrm{DAF}$, the number of oil bodies increased substantially and the oil bodies were scattered throughout the entire cell, which was very different from the images at 45 DAF. Whether the formation of the oil bodies is correlated with the rapid increase in contents of the three predominant UFAs requires further study.

Applications of MRI in seed science. Based on MRI, two significant seed development stages were captured. At 45 to 85 DAF, P. ostii seeds were in the morphological construction stage, during which biomass and oil content increased rapidly. During this stage, although the water content decreased sharply, the plants should be irrigated timeously to ensure adequate nutritional supply for seed growth. In addition, at this stage cell enlargement and expansion are active, so fertilization should be strengthened to meet the nutrient demands of the cells. Fazli et al. (2005) observed that fertilization can affect seed yield and oil content. However, no relevant research has been conducted on $P$. ostii previously. Therefore, the optimal amount and type of fertilization for $P$. ostii needs to be explored. At 85 to 115 DAF, $P$. ostii seeds entered the nutrient accumulation stage. Through accumulation of nutrients at earlier stages, the carbohydrates accumulated in the seeds were converted into insoluble polymers (such as lipids) for storage, and a portion of the carbohydrates were gradually converted to UFAs.
The magnetic resonance images showed that at $45 \mathrm{DAF}$, the proton density in the seed periphery near the seedcoat was greater than that in other areas of the seed. This may indicate that development of the internal components of the seeds was not complete, so the seed was filled circumferentially from the seedcoat to the central area of the endosperm. As the seeds developed, the magnetic resonance images showed that brightness was greater at $65 \mathrm{DAF}$ (Fig. 3D) than at $45 \mathrm{DAF}$ (Fig. 3B). The reason may be that, during the two stages, the water content decreased significantly, yet oil began to accumulate rapidly, and the hydrogen protons lost in water were fewer than the accumulated hydrogen protons in the oil. When the seeds entered the nutrient accumulation stage [see the pseudocolor image at $105 \mathrm{DAF}$ (Fig. 3J)], it was observed that the majority of the red areas in the follicles disappeared and the more yellow areas were present. The reason for the change may be that the water content of seeds in 

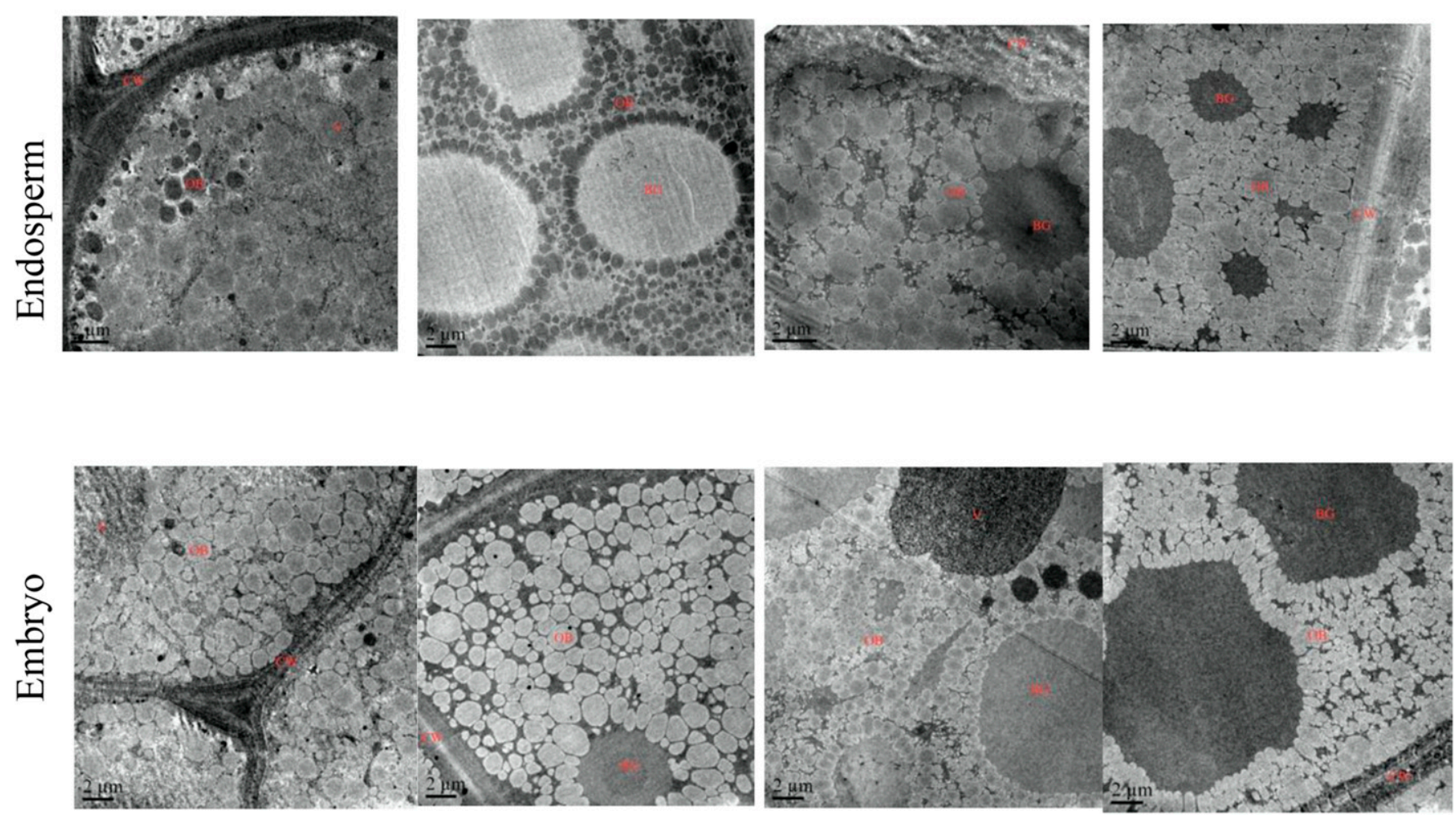

Fig. 4. Ultrastructure of endosperm and embryo cells of Paeonia ostii seeds at 45, 65, 85, and $115 \mathrm{~d}$ after flowering (DAF) visualized by transmission electron microscopy. $\mathrm{OB}$, oil body; $\mathrm{BG}$, highly electron-dense substance; $\mathrm{CW}$, cell wall; $\mathrm{N}$, nucleus.

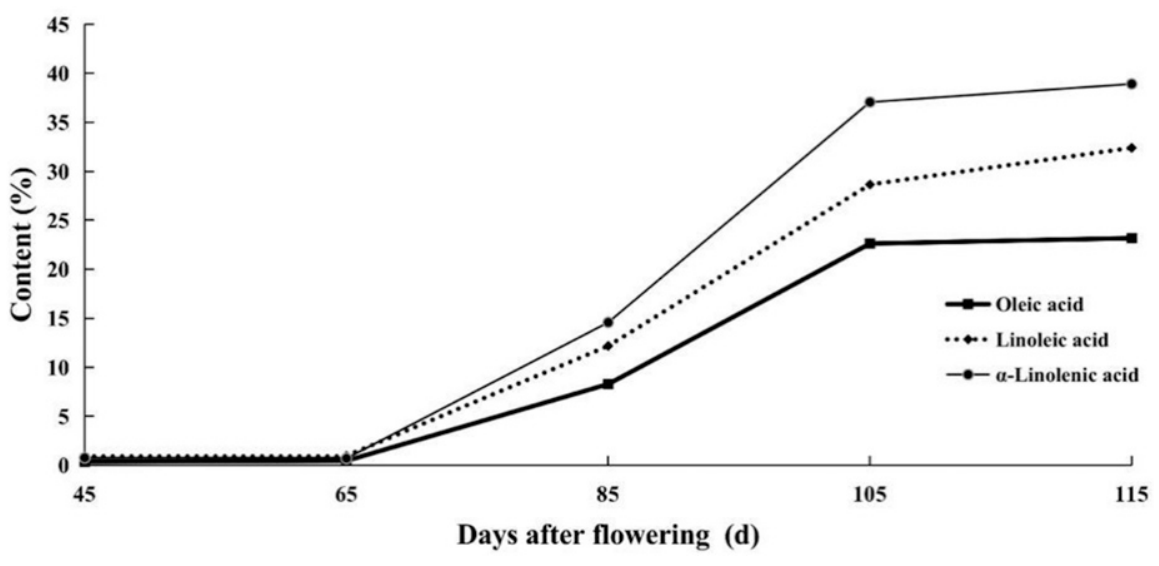

Fig. 5. Unsaturated fatty acid contents in Paeonia ostii seeds during follicle maturation.

P. ostii follicles decreased, and the degrees of proton freedom decreased (i.e., part of the free water was converted to bound water).

\section{Conclusions}

Based on the magnetic resonance images, the development of $P$. ostii seeds can be divided into two developmental stages: a morphological construction stage (45-85 DAF) and a nutrient accumulation stage (95-115 DAF). At the early stage of seed development (45 DAF), in the endosperm, a few oil bodies were scattered at the periphery of the cell wall, and the formation of oil bodies in the endosperm was delayed compared with that in the embryo. With progression in seed development, the number and size of oil bodies in the embryo and endosperm continued to grow. The oil content in $P$. ostii seeds continued to accumulate during the development process and peaked at 85 DAF, whereas the water content showed the opposite trend. Also at $85 \mathrm{DAF}$, the three predominant UFAs in $P$. ostii seeds showed a slow-fast-slow accumulation pattern, and all accumulated rapidly from 65 to 105 DAF, which represents a critical period for cultivation of $P$. ostii shrubs with a high $\alpha$-linolenic acid content.

\section{Literature Cited}

Cheng, F.Y. and X.N. Yu. 2008. Flare tree peonies (Paeonia rockii hybrids) and the origin of the cultivar group. Acta Hort. 766:375-382.

Dutta, R., U. Sarkar, and A. Mukherjee. 2014. Extraction of oil from Crotalaria juncea seeds in a modified Soxhlet apparatus: Physical and chemical characterization of a prospective biofuel. Fuel 116:794-802.

Fazli, I.S., M.Z. Abdin, A. Jamal, and S. Ahmad. 2005. Interactive effect of sulphur and nitrogen on lipid accumulation, acetyl-CoA concentration and acetyl-CoA carboxylase activity in developing seeds of oilseed crops (Brassica campestris L. and Eruca sativa Mill.). Plant Sci 168(1):1-36.

Fofana, B., S. Cloutier, S. Duguid, J. Ching, and C. Rampitsch. 2006. Gene expression of stearoylACP desaturase and $\Delta 12$ fatty acid desaturase 2 is modulated during seed development of flax (Linum usitatissimum). Lipids 41(7):705-712.

Gai, S., Y. Zhang, C. Liu, Y. Zhang, and G. Zheng. 2013. Transcript profiling of Paoenia ostii during artificial chilling induced dormancy release identifies activation of GA pathway and carbohydrate metabolism. PLoS One 8(2):e55297.

Han, J.G., Y. Song, Z.G. Liu, and Y.H. Hu. 2011. Culturable bacterial community analysis in the root domains of two varieties of tree peony (Paeonia ostii). FEMS Microbiol. Lett. 322(1): 15-24

Haralampidis, K., D. Milioni, J. Sanchez, M. Baltrusch, E. Heinz, and P. Hatzopoulos. 1998. Temporal and transient expression of stearoyl-ACP carrier protein desaturase gene during olive fruit development. J. Expt. Bot. 49(327):1661-1669. 
Li, Y.C. 2014. The strategy on the oil tree peony industry in China. China Eng. Sci. 16(10):5863. (In Chinese).

Li, X.Q., J.G. Han, Z. Liu, Q.H. Liu, and Y.H. Hu. 2014. Economic characteristics investigation and seed oil fatty acid composition analysis of Paeonia ostii plants in different areas. Cereals Oils. 4:47-50. (In Chinese).

Mou, H.M., J.Q. He, J.J. Xing, S.J. Yao, Y. Tang, Z.Q. Wang, and G.Y. Du. 2016. Water changes in wheat spike during grain filling stage investigated by nuclear magnetic resonance. Trans. Chinese Soc. Agr. Eng. 32(8):98-104. (In Chinese).

Pavithra, H.R., G. Balakrishna, and M.B. Shivanna. 2014. Biochemical changes in the composition of developing seeds of Pongamia pinnata (L.) Pierre. Ind. Crops Prod. 53:199-208.

Pavithra, H.R., B. Gowda, K.R. Kumar, K.T. Prasanna, and M.B. Shivanna. 2012. Oil, fatty acid profile and karanjin content in developing Pongamia pinnata (L.) Pierre seeds. J. Amer. Oil Chem. Soc. 89(12):2237-2244.
Prestes, R.A., L.A. Colnago, L.A. Forato, L. Vizzotto, E.H. Novotny, and E.A. Carrilho. 2007. Rapid and automated low resolution NMR method to analyze oil quality in intact oilseeds. Anal. Chim. Acta 596(2):325329.

Shi, S. 2016. Effects of intercropping and fertilization on the yield and quality of Paeonia ostii seeds. Anhui Agr. Univ., Anhui, master's thesis. (In Chinese).

Wiberg, E. and A.B. Stymne. 1997. Fatty acid distribution and lipid metabolism in developing seeds of laurate-producing rape (Brassica napus L.). Planta 203(3):341-348.

Yuan, J.H., F.Y. Cheng, and S.L. Zhou. 2011. The phylogeographic structure and conservation genetics of the endangered tree peony, Paeonia rockii (Paeoniaceae), inferred from chloroplast gene sequences. Conserv. Genet. 12(6):15391549.

Yuan, L.X., J.X. Hao, G.H. Zhou, Y. Sun, and R.Z. Li. 2015. Research progress on synthetic regulation of new energy crops, flaxseed and seed oils. Shanxi Agr. Sci. 5:634-638. (In Chinese).

Zarringhalami, S., M.A. Sahari, M. Barzegar, and Z. Hamidi-esfehani. 2011. Changes in oil content, chemical properties, fatty acid composition and triacylglycerol species of tea seed oil during maturity period. J. Food Biochem. 35(4):1161-1169.

Zhang, Z.H., Y. Luo, X. Wang, and F.Y. Yu. 2018. Quantitative spatiotemporal oil body ultrastructure helps to verify the distinct lipid deposition patterns in Benzoin endosperm and embryo cells. Forests 9(5):265.

Zhang, Z.H., X. Wang, Y. Luo, and F.Y. Yu. 2017. Carbon competition between fatty acids and starch during benzoin seeds maturation slows oil accumulation speed. Trees 31(3):10251039.

Zhao, C.G. 2010. Study on the rule of oil accumulation during seed development of Xanthoceras sorbifolium Bunge fruiting. Beijing Forestry Univ., Beijing, master's thesis. (In Chinese). 